\title{
Locking in the incompressible limit: pseudo-divergence-free element free Galerkin
}

\author{
Yolanda Vidal $^{1, *}$ Pierre Villon $^{2}$ and Antonio Huerta ${ }^{1, *}$ \\ ${ }^{1}$ Departament de Matemàtica Aplicada III, E.T.S. de Ingenieros de Caminos, Canales y Puertos, Universitat Politècnica de \\ Catalunya, Jordi Girona 1, E-08034 Barcelona, Spain. \\ ${ }^{2}$ Département Génie des systèmes mécaniques Université de Technologie de Compiègne, Compiègne, France.
}

\begin{abstract}
SUMMARY
Locking in finite elements has been a major concern since its early developments. It appears because poor numerical interpolation leads to an over-constrained system. This paper proposes a new formulation that asymptotically suppresses locking for the Element Free Galerkin (EFG) method in incompressible limit, i.e. the so-called volumetric locking. Originally it was claimed that EFG did not present volumetric locking. However, recently, performing a modal analysis, the senior author has shown that EFG presents volumetric locking. In fact, it is concluded that an increase of the dilation parameter attenuates, but never suppresses, the volumetric locking and that, as in standard finite elements, an increase in the order of reproducibility (interpolation degree) reduces the relative number of locking modes. Here an improved formulation of the Element Free Galerkin method is proposed in order to alleviate volumetric locking.

Diffuse derivatives are defined in the thesis of the second author and their convergence to the derivatives of the exact solution, when the radius of the support goes to zero (for a fixed dilation parameter), it's proved. Therefore diffuse divergence converges to the exact divergence. Since the diffuse divergence-free condition can be imposed a priori, new interpolation functions are defined that asymptotically verify the incompressibility condition. Modal analysis and numerical results for classical benchmark tests in solids corroborate this issue. Copyright (c) 2002 John Wiley \& Sons, Ltd.
\end{abstract}

KEY WORDS: Incompressible Locking, Element Free Galerkin, Meshless, Mesh-free, Diffuse derivatives.

\section{INTRODUCTION}

In a recent paper [1] locking of the Element Free Galerkin (EFG) method near the incompressible limit, i.e. the so-called volumetric locking, has been studied. In particular, the standard EFG behavior is compared with fi nite elements, bilinear and biquadratic interpolations. Locking of standard fi nite elements has been extensively studied and one can fi nd in the literature several remedies to suppress or at least alleviate locking [3].

\footnotetext{
* Correspondence to: Departament de Matemàtica Aplicada III, E.T.S. de Ingenieros de Caminos, Canales y Puertos, Universitat Politècnica de Catalunya, Jordi Girona 1, E-08034 Barcelona, Spain.

Contract/grant sponsor: Ministerio de Ciencia y Tecnología; contract/grant number: DPI2001-2204 and REN2001-0925-C03-01
} 
However, even recently [4] it was claimed that mesh-free methods did not exhibit volumetric locking. Now it is clear that this is not true. For instance, in [5] the numerical inf-sup condition is used to analyze the EFG method. Moreover, several authors have studied the influence of the dilation parameter in locking phenomena, either by numerical experiments [5, 6] or by heuristic arguments [7] based on the constraint ratio proposed by Hughes [3]. Now it is clear [1] that the dilation parameter attenuates locking but does not suppress the locking modes and that, as in fi nite elements, an increase in the order of consistency decreases the number of locking modes. The remedies proposed in the literature are extensions of the methods developed for fi nite elements $[5,7]$.

Here a novel approach is explored. It consist in using interpolation functions that verify approximately the divergence-free restriction. These interpolating functions can be defi ned a priori and are independent of the particle distribution. Moreover, as the density of particles is increased (i.e. as the discretization is refi ned) the divergence-free condition is better approximated. This method is based on diffuse derivatives [8], which converge to the derivatives of the exact solution when the radius of the support goes to zero (for a fi xed dilation parameter) [2].

\section{Diffuse derivatives}

\subsection{Preliminaries of the EFG method}

This section will not be devoted to develop or discuss mesh-free methods in detail or their relation with moving least squares (MLS) interpolants. There are well known references with excellent presentations of mesh-free methods, see for instance $[8,9,10,11,12]$. Here some basic notions will be recalled in order to introduce the notation and the approach employed in following sections.

The moving least squares approach is based on the local (i.e. at any point $z$ in the neighborhood of $\boldsymbol{x})$ approximation of the unknown scalar function $u(\boldsymbol{z})$ by $u^{\rho}$ as

$$
u(z) \simeq u^{\rho}(\boldsymbol{x}, \boldsymbol{z})=\mathbf{P}^{\top}(\boldsymbol{z}) \mathbf{a}(\boldsymbol{x}) \quad \text { for } \boldsymbol{z} \text { near } \boldsymbol{x}
$$

where the coeffi cients $\mathbf{a}(\boldsymbol{x})=\left\{\mathrm{a}_{\mathrm{b}}(\boldsymbol{x}), \mathrm{a}_{1}(\boldsymbol{x}), \ldots, \mathrm{a}_{l}(\boldsymbol{x})\right\}^{\top}$ are not constant, they depend on point $\boldsymbol{x}$, and $\mathbf{P}(\boldsymbol{z})=\left\{p_{0}(\boldsymbol{z}), p_{1}(\boldsymbol{z}), \ldots, p_{l}(\boldsymbol{z})\right\}^{\top}$ includes a complete basis of the subspace of polynomials of degree $m$. In one dimension, it is usual that $p_{i}(x)$ coincides with the monomials $x^{i}$, and, in this particular case, $l=m$. The coeffi cients a are obtained by the minimization of the functional $J_{\boldsymbol{x}}(\mathbf{a})$ centered in $\boldsymbol{x}$ and defi ned as

$$
J_{\boldsymbol{x}}(\mathbf{a})=\sum_{i \in I_{\boldsymbol{x}}} \phi\left(\boldsymbol{x}, \boldsymbol{x}_{i}\right)\left[u\left(\boldsymbol{x}_{i}\right)-\mathbf{P}\left(\boldsymbol{x}_{i}\right) \mathbf{a}(\boldsymbol{x})\right]^{2}
$$

where $\phi\left(\boldsymbol{x}, \boldsymbol{x}_{i}\right)$ is a weighting function (positive, even and with compact support) which characterizes the mesh-free method. For instance, if $\phi\left(\boldsymbol{x}, \boldsymbol{x}_{i}\right)$ is continuous together with its fi rst $k$ derivatives, the interpolation is also continuous together with its first $k$ derivatives. The particles cover the computational domain $\Omega, \Omega \subset \mathbb{R}^{\mathrm{n}_{\text {sd }}}$, and in particular a number of particles $\left\{\boldsymbol{x}_{i}\right\}_{i \in I_{\boldsymbol{x}}}$ belong to the support of $\phi\left(\boldsymbol{x}, \boldsymbol{x}_{i}\right)$. The minimization of $J_{\boldsymbol{x}}(\mathbf{a})$ induces the standard normal equations in a weighted least squares problem

$$
\mathbf{M}(\boldsymbol{x}) \mathbf{a}(\boldsymbol{x})=\sum_{i \in I_{\boldsymbol{x}}} \phi\left(\boldsymbol{x}, \boldsymbol{x}_{i}\right) u\left(\boldsymbol{x}_{i}\right) \mathbf{P}\left(\boldsymbol{x}_{i}\right)
$$

where, as usual, the Gram matrix $\mathbf{M}(\boldsymbol{x})$ is the scalar product of the interpolation polynomials:

$$
\mathbf{M}(\boldsymbol{x})=\sum_{i \in I_{\boldsymbol{x}}} \phi\left(\boldsymbol{x}, \boldsymbol{x}_{i}\right) \mathbf{P}\left(\boldsymbol{x}_{i}\right)^{\top} \mathbf{P}\left(\boldsymbol{x}_{i}\right) .
$$


That is,

$$
<u, v>=\sum_{i \in I_{\boldsymbol{x}}} \phi\left(\boldsymbol{x}, \boldsymbol{x}_{i}\right) u\left(\boldsymbol{x}_{i}\right) v\left(\boldsymbol{x}_{i}\right)
$$

must defi ne a discrete scalar product. Thus, several conditions on the particle distribution are implicitly assumed, see for instance [13].

Once the normal equations are solved (3) the coeffi cients a are substituted in (1). Since the weighting function $\phi$ usually favors the central point $\boldsymbol{x}$, it seems reasonable to assume that such an approximation is more accurate precisely at $\boldsymbol{z}=\boldsymbol{x}$ and thus the approximation (1) is particularized at $\boldsymbol{x}$, that is,

$$
u(\boldsymbol{x}) \simeq u^{\rho}(\boldsymbol{x})=\mathbf{P}^{\top}(\boldsymbol{x}) \mathbf{a}(\boldsymbol{x})=\mathbf{P}^{\top}(\boldsymbol{x}) \mathbf{M}^{-1}(\boldsymbol{x}) \sum_{i \in I_{\boldsymbol{x}}} \phi\left(\boldsymbol{x}, \boldsymbol{x}_{i}\right) u\left(\boldsymbol{x}_{i}\right) \mathbf{P}\left(\boldsymbol{x}_{i}\right) .
$$

This expression can also be written in a standard interpolation form

$$
u^{\rho}(\boldsymbol{x})=\sum_{i \in I_{\boldsymbol{x}}} N_{i}^{\rho}(\boldsymbol{x}) u\left(\boldsymbol{x}_{i}\right)=\sum_{i \in I_{\boldsymbol{x}}}\left[\phi\left(\boldsymbol{x}, \boldsymbol{x}_{i}\right) \mathbf{P}^{\top}(\boldsymbol{x}) \mathbf{M}^{-1}(\boldsymbol{x}) \mathbf{P}\left(\boldsymbol{x}_{i}\right)\right] u\left(\boldsymbol{x}_{i}\right)
$$

\subsection{The diffuse derivative}

The approximation of the derivative of $u$ is the derivative of $u^{\rho}$. This requires to derive (6), that is

$$
\frac{\partial u}{\partial x_{i}} \simeq \frac{\partial u^{\rho}}{\partial x_{i}}=\frac{\partial \mathbf{P}^{\top}}{\partial x_{i}} \mathbf{a}(\boldsymbol{x})+\mathbf{P}^{\top} \frac{\partial \mathbf{a}}{\partial x_{i}} \quad \text { for } i=1, \ldots, \mathrm{n}_{\mathrm{sd}} .
$$

Note that the derivative of the polynomials in $\mathbf{P}$ is trivial but the derivative of the coeffi cients a requires the resolution of a linear system of equations with the same matrix $\mathbf{M}$, see [14]. Moreover, the derivatives of the polynomials can be done a priori but the derivatives of the coeffi cients require the knowledge of the cloud of particles surrounding each point $\boldsymbol{x}$.

Thus the concept of diffuse derivative, see $[2,8]$, defi ned as

$$
\frac{\delta u^{\rho}}{\delta x_{i}}=\left.\frac{\partial u^{\rho}}{\partial z_{i}}\right|_{\boldsymbol{z}=\boldsymbol{x}}=\left.\frac{\partial \mathbf{P}^{\top}}{\partial z_{i}}\right|_{\boldsymbol{z}=\boldsymbol{x}} \mathbf{a}(\boldsymbol{x})=\frac{\partial \mathbf{P}^{\top}}{\partial x_{i}} \mathbf{a}(\boldsymbol{x}) \quad \text { for } i=1, \ldots, \mathrm{n}_{\mathrm{sd}}
$$

is from a computational cost point of view an interesting alternative to (8). Moreover, the diffuse derivative converges at optimal rate to the derivative of $u$, see the demonstration in [2]. For an approximation $u^{\rho}$ to $u$ with an order of consistency $m$ (i.e. $\mathbf{P}$ includes a complete base of the subspace of polynomials of degree $m$ ), then

$$
\left\|\frac{\partial^{k} u}{\partial x_{i}^{k}}-\frac{\delta^{k} u^{\rho}}{\delta x_{i}^{k}}\right\|_{\infty} \leq C(x) \frac{\rho^{m+1-k}}{(m+1) !} \quad \forall k=0, \ldots, m \quad \text { and for } i=1, \ldots, \mathrm{n}_{\mathrm{sd}} .
$$

\section{Pseudo-divergence free condition}

\subsection{Diffuse divergence}

In the previous section the diffuse derivative was introduced and it was recalled that it converges to the actual derivative as $\rho \rightarrow 0$. Moreover, diffuse derivatives only act on the polynomials $\mathbf{P}$. Incompressible computations require that the approximating fi eld must be divergence free. That is, the solution $\boldsymbol{u}(\boldsymbol{x})$, now a vector $\boldsymbol{u}: \mathbb{R}^{\mathrm{n}_{\text {sd }}} \rightarrow \mathbb{R}^{\mathrm{n}_{\mathrm{sd}}}$, verifi es $\nabla \cdot \boldsymbol{u}=0$, and the approximation $\boldsymbol{u}(\boldsymbol{x})$ should 
also be divergence-free. This condition however depends on the interpolation space. Here, instead of requiring a divergence-free interpolation, the diffuse divergence of the approximation

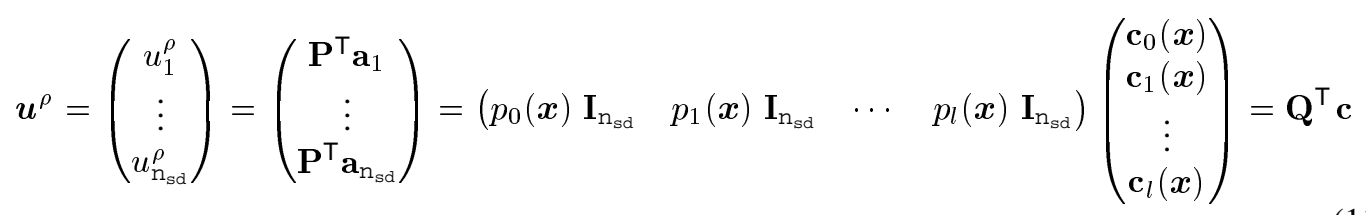

is imposed equal to zero, i.e.

$$
\nabla^{\delta} \cdot \boldsymbol{u}^{\rho}=\sum_{i=1}^{\mathrm{n}_{\text {sd }}} \frac{\delta \boldsymbol{u}^{\rho}}{\delta x_{i}}=\sum_{i=1}^{\mathrm{n}_{\text {sd }}} \frac{\partial \mathbf{P}^{\top}}{\partial x_{i}} \mathbf{a}_{i}(\boldsymbol{x})=\left(\nabla \cdot \mathbf{Q}^{\top}(\boldsymbol{x})\right) \mathbf{c}(\boldsymbol{x})=0 .
$$

Note that $\mathbf{I}_{\mathrm{n}_{\mathrm{sd}}}$ is the identity matrix of order $\mathrm{n}_{\mathrm{sd}}$ and the coeffi cients have been rearranged as

$$
\mathbf{c}^{\top}=(\underbrace{\mathrm{a}_{0,1} \cdots \mathrm{a}_{0, \mathrm{n}_{\text {sd }}}}_{\mathbf{c}_{0}^{\top}(\boldsymbol{x})} \underbrace{\mathrm{a}_{1,1} \cdots \mathrm{a}_{1, \mathrm{n}_{\mathrm{sd}}}}_{\mathbf{c}_{1}^{\top}(\boldsymbol{x})} \cdots \underbrace{\mathrm{a}_{l, 1} \cdots \mathrm{a}_{l, \mathrm{n}_{\text {sd }}}}_{\mathbf{c}_{l}^{\top}(\boldsymbol{x})}) .
$$

Equation (12) must hold at each point $\boldsymbol{x}$ and for any approximation. Thus appropriate interpolation functions, $\mathbf{Q}$, must be defi ned in order to verify (12) and thus ensure asymptotically a divergence-free interpolation (i.e. the divergence-free condition is fulfi lled as $\rho \rightarrow 0$ ).

\subsection{A 2D pseudo-divergence free interpolation}

The previous concepts are particularized for a two-dimensional case and allow to defi ne the pseudodivergence-free interpolation functions. Suppose for instance that consistency of order two is desired, then $\mathbf{P}^{\top}=\left\{1, x_{1}, x_{2}, x_{1}^{2}, x_{1} x_{2}, x_{2}^{2}\right\}$, thus

and

$$
\mathbf{Q}^{\top}=\left(\begin{array}{cccccccccccc}
1 & 0 & x_{1} & 0 & x_{2} & 0 & x_{1}^{2} / 2 & 0 & x_{1} x_{2} & 0 & x_{2}^{2} / 2 & 0 \\
0 & 1 & 0 & x_{1} & 0 & x_{2} & 0 & x_{1}^{2} / 2 & 0 & x_{1} x_{2} & 0 & x_{2}^{2} / 2
\end{array}\right)
$$

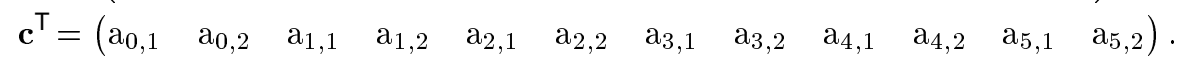

The pseudo-divergence-free condition (12) is, in this case, written as

$$
\nabla^{\delta} \cdot \boldsymbol{u}^{\rho}=\frac{\partial \mathbf{P}^{\top}}{\partial x_{1}} \mathbf{a}_{1}+\frac{\partial \mathbf{P}^{\top}}{\partial x_{2}} \mathbf{a}_{2}=0
$$

which implies

and consequently,

$$
\begin{aligned}
& \left(\mathrm{a}_{1,1}+\mathrm{a}_{2,2}\right)+x_{1}\left(\mathrm{a}_{3,1}+\mathrm{a}_{4,2}\right)+x_{2}\left(\mathrm{a}_{4,1}+\mathrm{a}_{5,2}\right)=0, \\
& \mathrm{a}_{1,1}+\mathrm{a}_{2,2}=0, \quad \mathrm{a}_{3,1}+\mathrm{a}_{4,2}=0, \quad \mathrm{a}_{4,1}+\mathrm{a}_{5,2}=0 .
\end{aligned}
$$

The influence of these three restrictions in the interpolation functions (14) can be viewed as follows

$$
\left(\begin{array}{cccccccccccc}
1 & 0 & x_{1} & 0 & x_{2} & 0 & x_{1}^{2} / 2 & 0 & x_{1} x_{2} & 0 & x_{2}^{2} / 2 & 0 \\
0 & 1 & -x_{2} & x_{1} & 0 & 0 & -x_{1} x_{2} & x_{1}^{2} / 2 & -x_{2}^{2} / 2 & 0 & 0 & 0
\end{array}\right),
$$

where one should note that the coeffi cients in the $x_{1}$ and $x_{2}$ directions are now coupled and that the total number of degrees of freedom has decreased. 


\subsection{The pseudo-divergence-free EFG method}

Using (19), let $\mathbf{Q}_{\delta}$ be the new interpolation matrix (where obviously the unnecessary columns have been removed). The interpolation is then defi ned as

$$
\boldsymbol{u}(\boldsymbol{z}) \simeq \boldsymbol{u}^{\rho}(\boldsymbol{x}, \boldsymbol{z})=\left(\begin{array}{c}
u_{1}^{\rho}(\boldsymbol{x}, \boldsymbol{z}) \\
u_{2}^{\rho}(\boldsymbol{x}, \boldsymbol{z})
\end{array}\right)=\mathbf{Q}_{\delta}^{\top}(\boldsymbol{z}) \mathbf{c}(\boldsymbol{x}) .
$$

The vector version of the discrete scalar product defi ned in (5),

$$
<\boldsymbol{u}, \boldsymbol{v}>=\sum_{i \in I_{\boldsymbol{x}}} \phi\left(\boldsymbol{x}, \boldsymbol{x}_{i}\right) \boldsymbol{u}^{\top}\left(\boldsymbol{x}_{i}\right) \boldsymbol{v}\left(\boldsymbol{x}_{i}\right)
$$

allows now to reproduce the MLS approximation. Thus at each point $\boldsymbol{x}$ the normal equations should be solved, see (3),

$$
\mathbf{M}(\boldsymbol{x}) \mathbf{c}(\boldsymbol{x})=<u, \mathbf{Q}_{\delta}>\quad \text { with } \mathbf{M}(\boldsymbol{x}):=<\mathbf{Q}_{\delta}, \mathbf{Q}_{\delta}>.
$$

Thus, as previously, the coeffi cients $\mathbf{c}$ are substituted in (20) and the approximation is particularized at $\boldsymbol{z}=\boldsymbol{x}$. Then, equation (6) becomes

$$
\boldsymbol{u}(\boldsymbol{x}) \simeq \boldsymbol{u}^{\rho}(\boldsymbol{x})=\mathbf{Q}_{\delta}^{\top}(\boldsymbol{x}) \mathbf{c}(\boldsymbol{x})=\mathbf{Q}_{\delta}^{\top}(\boldsymbol{x}) \mathbf{M}^{-1}(\boldsymbol{x})<u, \mathbf{Q}_{\delta}>,
$$

and a fi nal expression similar to (7) can be found as

$$
\boldsymbol{u}^{\rho}(\boldsymbol{x})=\sum_{i \in I_{\boldsymbol{x}}} \mathbf{N}_{i}^{\rho}(\boldsymbol{x}) \boldsymbol{u}\left(\boldsymbol{x}_{i}\right)=\sum_{i \in I_{\boldsymbol{x}}}\left[\phi\left(\boldsymbol{x}, \boldsymbol{x}_{i}\right) \mathbf{Q}_{\delta}^{\top}(\boldsymbol{x}) \mathbf{M}^{-1}(\boldsymbol{x}) \mathbf{Q}_{\delta}\left(\boldsymbol{x}_{i}\right)\right] \boldsymbol{u}\left(\boldsymbol{x}_{i}\right) .
$$

It is important to note that the matrix of interpolation functions $\mathbf{N}_{i}^{\rho}$ is now a full matrix not a diagonal one as standard EFG would induce in this non scalar problem. This is due to the fact that the two components of the solution are linked by the incompressibility restriction.

\section{Modal analysis}

\subsection{Preliminaries}

The modal analysis presented here follows the same rationale originally presented in [1]. It is restricted to small deformations, namely $\boldsymbol{\nabla}^{\mathrm{s}} \boldsymbol{u}$, where $\boldsymbol{u}$ is the displacement and $\boldsymbol{\nabla}^{\mathrm{s}}$ the symmetric gradient, i.e. $\nabla^{\mathrm{s}}=\frac{1}{2}\left(\nabla^{T}+\nabla\right)$. Moreover, linear elastic isotropic materials under plane strain conditions are considered. Dirichlet boundary conditions are imposed on $\Gamma_{D}$, a traction $\boldsymbol{h}$ is prescribed along the Neumann boundary $\Gamma_{N}$ and there is a body force $f$. Thus, the problem that needs to be solved may be stated as: solve for $\boldsymbol{u} \in\left[H_{\Gamma_{D}}^{1}\right]^{2}$ such that

$$
\begin{aligned}
\frac{E}{1+\nu} \int_{\Omega} \boldsymbol{\nabla}^{\mathrm{s}} \boldsymbol{v}: \boldsymbol{\nabla}^{\mathrm{s}} \boldsymbol{u} d \Omega+\frac{E \nu}{(1+\nu)(1-2 \nu)} & \int_{\Omega}(\boldsymbol{\nabla} \cdot \boldsymbol{v})(\boldsymbol{\nabla} \cdot \boldsymbol{u}) d \Omega \\
& =\int_{\Omega} \boldsymbol{f} \cdot \boldsymbol{v} d \Omega+\int_{\Gamma_{N}} \boldsymbol{h} \cdot \boldsymbol{v} d \Gamma \quad \forall \boldsymbol{v} \in\left[H_{0, \Gamma_{D}}^{1}\right]^{2} .
\end{aligned}
$$

In this equation, the standard vector subspaces of $H^{1}$ are employed for the solution $\boldsymbol{u}$

$$
\left[H_{\Gamma_{D}}^{1}\right]^{2}:=\left\{\boldsymbol{u} \in\left[H^{1}\right]^{2} \mid \boldsymbol{u}=\boldsymbol{u}_{D} \text { on } \Gamma_{D}\right\}
$$


(Dirichlet conditions, $\boldsymbol{u}_{D}$, are automatically satisfi ed) and for the test functions $\boldsymbol{v}$

$$
\left[H_{0, \Gamma_{D}}^{1}\right]^{2}:=\left\{\boldsymbol{v} \in\left[H^{1}\right]^{2} \mid \boldsymbol{v}=\mathbf{0} \text { on } \Gamma_{D}\right\}
$$

(zero values are imposed along $\Gamma_{D}$ ).

This equation shows the inherent diffi culties of the incompressible limit. The standard a priori error estimate emanating from (25) and based on the energy norm, which is induced by the LHS of (25), is

$$
\left\|\boldsymbol{u}-\boldsymbol{u}_{h}\right\| \leq \inf _{\boldsymbol{w} \in \mathcal{S}_{h}}\|\boldsymbol{u}-\boldsymbol{w}\| \leq C_{\boldsymbol{u}, \nu, p} h^{f(p)}
$$

where $\mathcal{S}_{h}$ is the fi nite dimensional subspace of $\left[H_{\Gamma_{D}}^{1}\right]^{2}$ in which the approximation $\boldsymbol{u}_{h}$ is sought, $C_{\boldsymbol{u}, \nu, p}$ is a constant independent of $h$ (characteristic size of the mesh), and $f(p)$ is a positive monotone function of $p$ (degree of the polynomials used for the interpolation). The subindices of the constant $C$ indicate that it depends on the Poisson ratio, the order of the interpolation and the exact solution itself.

From (25) one can observe the diffi culties of the energy norm to produce a small infi mum in (26) for values of $\nu$ close to 0.5 . In fact, in order to have fi nite values of the energy norm the divergence-free condition must be enforced in the continuum case, i.e. $\boldsymbol{\nabla} \cdot \boldsymbol{u}=0$ for $\boldsymbol{u} \in\left[H_{\Gamma_{D}}^{1}\right]^{2}$, and also in the fi nite dimensional space, i.e. $\boldsymbol{\nabla} \cdot \boldsymbol{u}_{h}=0$ for $\boldsymbol{u}_{h} \in \mathcal{S}_{h} \subset\left[H_{\Gamma_{D}}^{1}\right]^{2}$. In fact, locking will occur when the approximation space $\mathcal{S}_{h}$ is not rich enough for the approximation to verify the divergence-free condition.

Under these conditions, it is evident that locking may be studied from the LHS of (25). This is the basis for the modal analysis of locking. The discrete eigenfunctions (the eigenvectors) corresponding to the LHS of (25) are computed because they completely describe, in the corresponding space, the behavior of the bilinear operator induced by this LHS.

In computational mechanics it is standard to write the strain, $\varepsilon$, and the stress, $\sigma$, tensors in vector form. Moreover, under the assumptions already discussed, they are related as

$$
\varepsilon=\mathbf{B d}, \quad \boldsymbol{\sigma}=\mathbf{C} \varepsilon, \quad \mathbf{C}=\frac{E}{(1+\nu)(1-2 \nu)}\left(\begin{array}{ccc}
1-\nu & \nu & 0 \\
\nu & 1-\nu & 0 \\
0 & 0 & \frac{1-2 \nu}{2}
\end{array}\right) .
$$

Where $\mathbf{d}$ is the vector of nodal displacements (the coeffi cients corresponding to the approximation $\boldsymbol{u}_{h}$ in the base of $\mathcal{S}_{h}$ ), and $\mathbf{B}$ is the standard matrix relating displacements and strains. Then, the stiffness matrix can be computed as usual,

$$
\mathbf{K}=\int_{\Omega} \mathbf{B}^{T} \mathbf{C B} d \Omega
$$

The modal analysis presented in the following is based on $\mathbf{K}$, which is naturally related to the energy norm in the fi nite dimensional interpolation space, $\mathcal{S}_{h}$, defi ned by the fi nite elements employed (and characterized by $\mathbf{B}$ ).

\subsection{Comparing EFG and pseudo-divergence-free EFG}

The incompressible limit is studied by evaluating the eigenvalues associated to each mode as the Poisson ratio, $\nu$, tends to 0.5 . As in [1] the logarithm of the eigenvalue is plotted as a function of the logarithm of $0.5-\nu$. Then each mode is classifi ed in three groups: (1) modes that do not present any locking behavior, (2) modes that do have physical locking, i.e. the eigenvalue goes to infi nity because it is a volumetric mode, and (3) modes associated to non-physical locking, i.e. the eigenvalue goes to infi nity but there is no volume variation. In the latter case, the displacement fi eld conserves the 


\section{Non-locking \\ Physical locking \\ Non-physical locking}
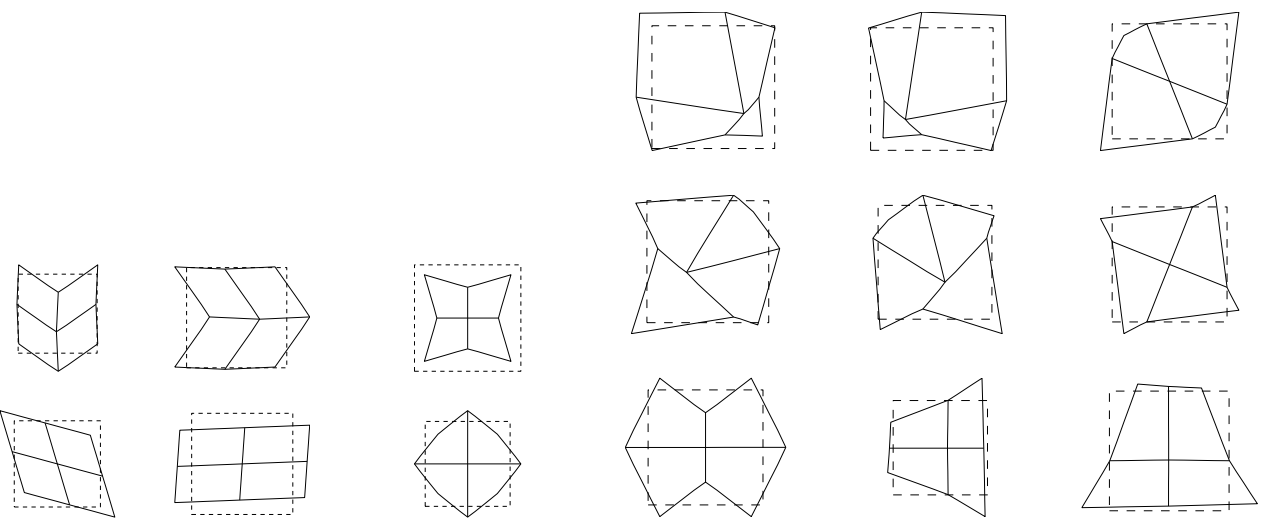

Figure 1. Modes for a $3 \times 3$ distribution of particles with bilinear consistency and $\rho / h=1.2$.

Non-locking
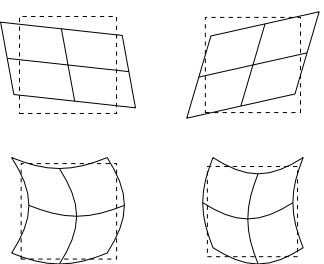

Physical locking
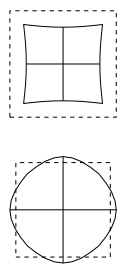

Non-physical locking
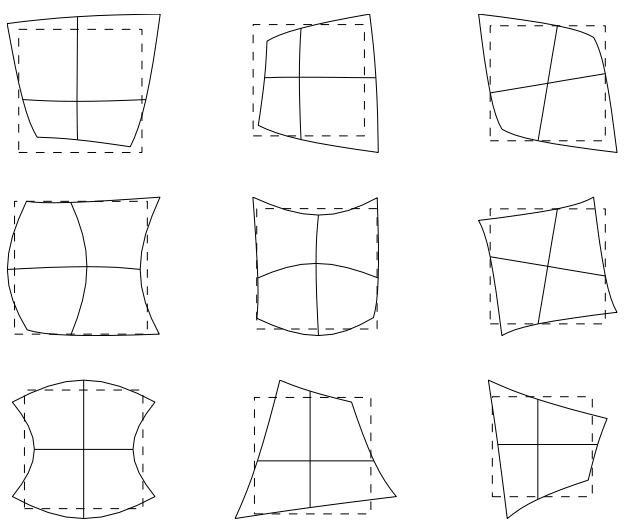

Figure 2. Modes for a $3 \times 3$ distribution of particles with bilinear consistency and $\rho / h=2.2$.

total area but suffers from non-physical locking. The interpolation space is not rich enough to ensure the divergence-free condition. In fact these modes do verify that

$$
\int_{\square} \nabla \cdot u_{h} d x=0,
$$

but do not comply with the local divergence-free condition. This is clearly a non-physical locking behavior.

The modal analysis is performed on a distribution of $3 \times 3$ particles and for bilinear consistency, i.e. $\mathbf{P}=\left\{1, x_{1}, x_{2}, x_{1} x_{2}\right\}^{\top}$. Figures 1 and 2 show the modes already classifi ed for two different dilation parameters, $\rho / h=1.2$ and 2.2. And Figure 3 compares the eigenvalues obtained by standard EFG and the pseudo-divergence-free interpolation for two particular non-physical locking modes with $\rho / h=1.2,2.2$ and 3.2. Note fi rst that the pseudo-divergence-free interpolation has not suppresses 

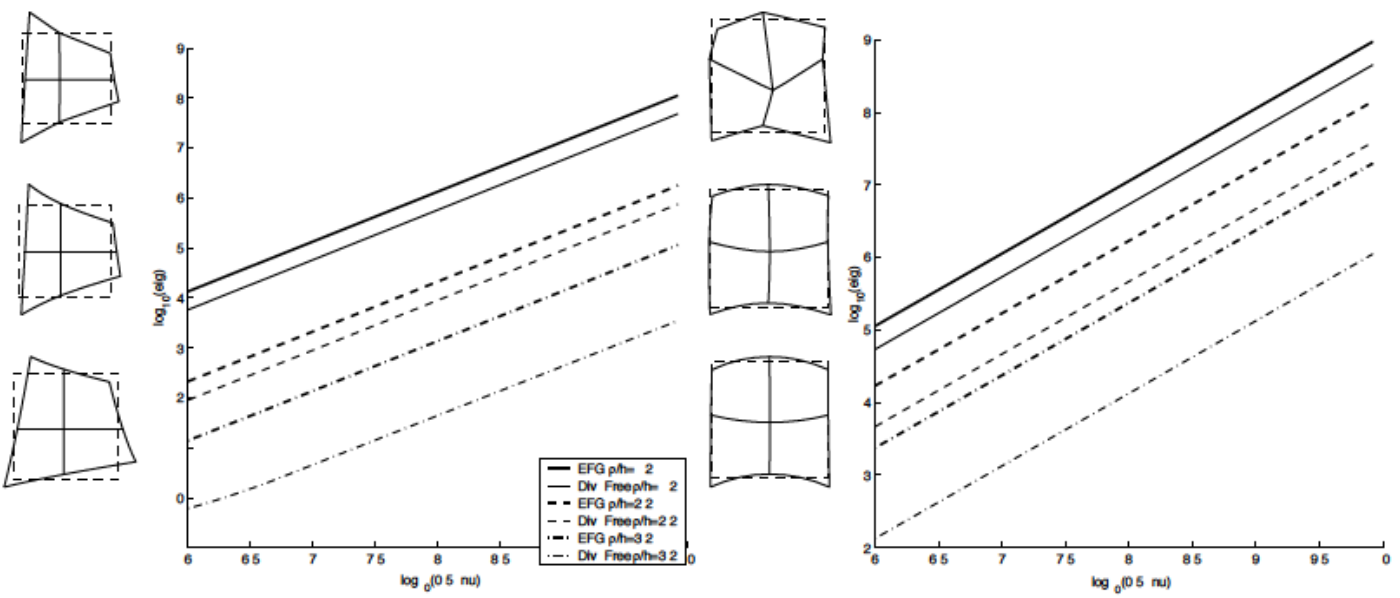

Figure 3. Variation of the eigenvalue as $\nu$ goes to 0.5 for the same non-physical locking mode with $\rho / h=1.2$, 2.2 and $3.2, \mathrm{EFG}$ results and pseudo-divergence-free interpolations.

the non-physical locking modes. Thus, for a fi xed dilation parameter $\rho$ variations on the ratio $\rho / h$ do not suppress locking. Indeed, the influence of locking is reduced because the eigenvalue is decreased. That is, the energy associated to the locking mode is decreased and this attenuates the volumetric locking. Nevertheless, in the incompressible limit locking will still be present and it may induce useless

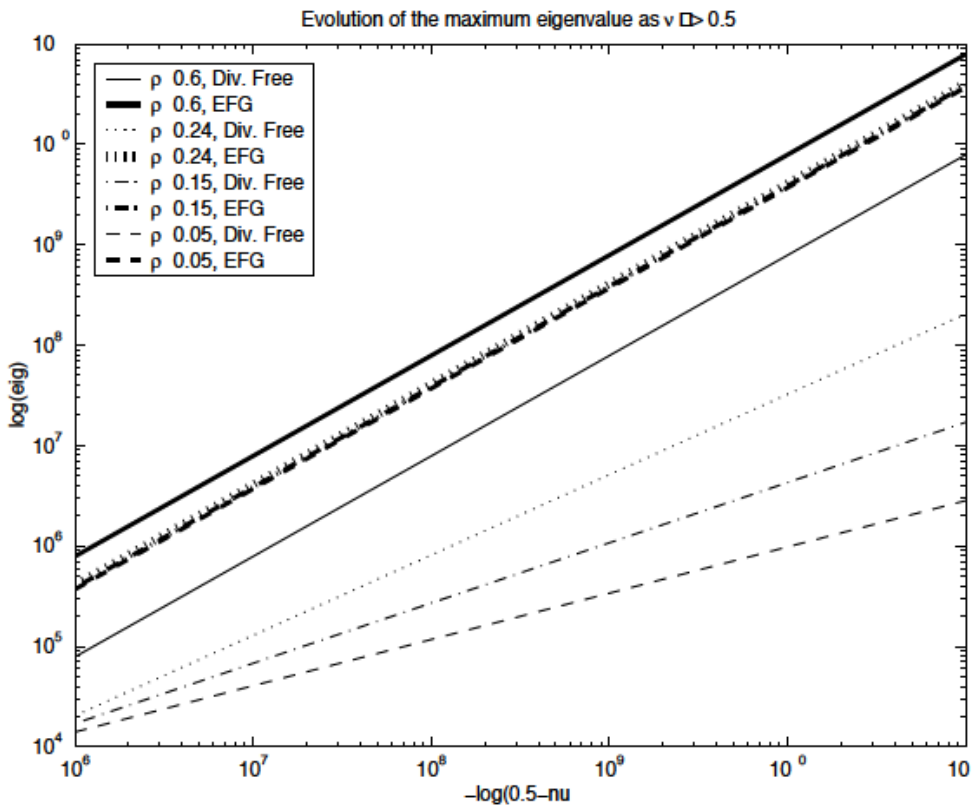

Figure 4. Variation of the maximum eigenvalue as $\nu$ goes to 0.5 , EFG results and pseudo-divergence-free interpolations. 
numerical results.

This results should be expected. The convergence of the diffuse derivative see (10) is ensured as $\rho$ approaches zero for a ratio $\rho / h$ kept constant. In other words, convergence is ensured as the interpolation is refi ned. This is analyzed in Figure 4 for the non-physical locking mode that presents the largest eigenvalue (the first mode to spoil the approximation). This results are obtained for the "worst" dilation parameter, $\rho / h=1.2$, that is the one that induces results more similar to bilinear fi nite elements. Three different values of $\rho$ are tested, $\rho=0.60,0.24$ and 0.15 . It is important to note that as $\rho$ decreases the eigenvalue also decreases (and drastically, the scale is logarithmic). Thus, as $\rho$ decreases the influence of locking attenuates. Moreover and more importantly, the slope of the curve also decreases as $\rho$ goes to zero (note that for standard EFG the slope remains constant). Thus, in the limit, as expected, the interpolation is divergence-free. Note that for $\rho=0.15$ and $\nu=0.5-10^{-11}$ the eigenvalue has been reduced in more than three orders of magnitude.

\section{Numerical examples}

\subsection{The cantilever beam}

A beam with linear isotropic material, under plane strain conditions and with a parabolic traction applied to the free end is considered, as shown in Figure 5. Displacements in both directions are prescribed at $\Gamma_{D}$. The prescribed displacements and the applied traction are such that the solution is known $[3,5]$ :

$$
\begin{gathered}
u_{1}=-2 \frac{1-\nu^{2}}{E} y\left[\left(48-3 x_{1}\right) x_{1}+\left(2+\frac{\nu}{1-\nu}\right)\left(x_{2}^{2}-0.25\right)\right], \\
u_{2}=2 \frac{1-\nu^{2}}{E}\left[3 \frac{\nu}{1-\nu} x_{2}^{2}\left(8-x_{1}\right)+\left(4+5 \frac{\nu}{1-\nu}\right) \frac{x_{1}}{4}+\left(24-x_{1}\right) x_{1}^{2}\right] \\
\sigma_{11}=-12 x_{2}\left(8-x_{1}\right), \quad \sigma_{22}=0, \quad \sigma_{12}=6\left(0.25-x_{2}^{2}\right)
\end{gathered}
$$

The problem is solved with uniform distributions of particles. Figure 6 shows the relative $L_{2}$ error in

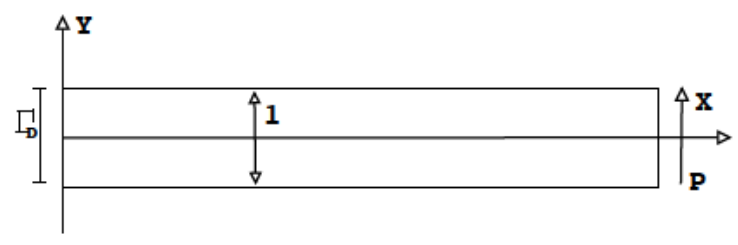

Figure 5. Cantilever beam problem

displacements for $\nu=0.3,0.5-10^{-4}$ and $0.5-10^{-6}$. Results are shown for bilinear consistency and $\rho / h=2.2$. The EFG results are compared with the pseudo-divergence-free interpolation. For EFG the typical convergence rates are obtained when $\nu=0.3$, but, as expected, results degrade as $\nu$ approaches the incompressible limit 0.5 . However, the pseudo-divergence free interpolation is able to reproduce the theoretical rate of convergence even for a nearly incompressible case $\nu=0.5-10^{-6}$ and a moderately fi ne discretization $(h<0.25$, i.e. $\rho<0.55)$. 


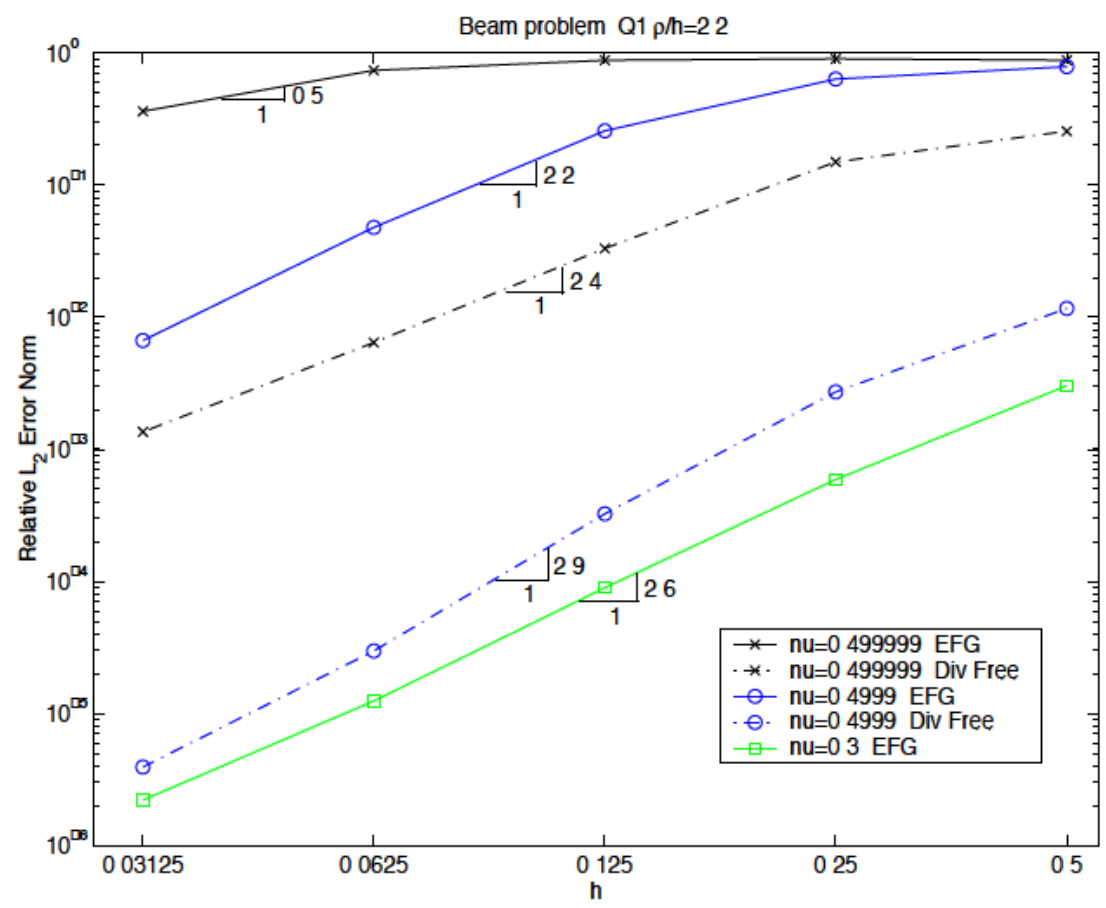

Figure 6. Cantilever beam with bilinear consistency and $\rho / h=2.2$

\section{Conclusions}

An improved formulation of the Element Free Galerkin method is proposed in order to alleviate volumetric locking. It is based on a pseudo-divergence-free interpolation, which asymptotically verifi es the incompressibility condition and is defi ned a priori. This interpolation is obtained imposing a zero diffuse divergence. Diffuse derivatives converge to the derivatives of the exact solution when the discretization is refi ned. Therefore the diffuse divergence converges to the exact divergence. Modal analysis and a numerical results for a classical benchmark tests in solids corroborate this issue.

\section{REFERENCES}

1. Huerta A, Fernández-Méndez S. Locking in the incompressible limit for the Element Free Galerkin method International Journal for Numerical Methods in Engineering 2001; 51(11):1361-1383.

2. Villon P. Contribution à l'optimisation Thèse présentée pour 1'obtention du grade de Docteur d'État, Université de Technologie de Compiègne, France, 1991.

3. Hughes TJR. The Finite Element Method, Linear Static and Dynamic Analysis Prentice-Hall, Englewood Cliffs, New Jersey, 1987

4. Zhu T, Atluri SN. A modifi ed collocation method and a penalty formulation for enforcing the essential boundary conditions in the element free Galerkin method Computational Mechanics 1998; 21:211-222.

5. Dolbow J, Belytschko T. Volumetric locking in the Element Free Galerkin method International Journal for Numerical Methods in Engineering 1999; 46:925-942. 
6. Askes H, de Borst R, Heeres O. Conditions for locking-free elasto-plastic analyses in the Element-Free Galerkin method Computer Methods in Applied Mechanics and Engineering 1999; 173:99-109.

7. Chen J, Yoon S, Wang H, Liu WK. Computer Methods in Applied Mechanics and Engineering 2000; 181:117-145.

8. Nayroles B, Touzot G, Villon P. Generating the fi nite element method: diffuse approximation and diffuse elements Computational Mechanics 1992; 10:307-318

9. Belytschko T, Krongauz Y, Organ D, Fleming M, Krysl P. Meshless Methods: an Overview and Recent Developments Computer Methods in Applied Mechanics and Engineering 1996; 139:3-47.

10. Liu WK, Belytschko T, Oden JT, editors. Meshless Methods Computer Methods in Applied Mechanics and Engineering 1996; 139

11. Liu WK, Chen Y, Jun S, Chen JS, Belytschko T, Pan C, Uras RA, Chang CT. Overview and Applications of the Reproducing Kernel Particle Methods Archives of Computational Methods in Engineering, State of the Art Reviews 1996; 3:3-80.

12. Liu WK, Li S, Belytschko T. Moving least square reproducing kernel methods. (I) Methodology and convergence Computer Methods in Applied Mechanics and Engineering 2000; 143:113-154.

13. Huerta A, Fernández-Méndez S. Enrichment and coupling of the fi nite element and meshless methods International Journal for Numerical Methods in Engineering 2000; 48:1615-1636.

14. Belytschko T, Krongauz Y, Fleming M, Organ D, Liu WK. Smoothing and Accelerated Computations in the Element Free Galerkin Method Journal of Computational and Applied Mathematics 1996; 74:111-126. 